\title{
Constant-Power Adaptive Orthogonally Multiplexed Modulations Under Flat Rayleigh Fading
}

\author{
Wei-Lun Lin and Char-Dir Chung
}

\begin{abstract}
Adaptive transmission technique adopting rectangularly-pulsed orthogonally multiplexed modulation (OMM) signals is studied for the flat Rayleigh fading channel. By choosing spectrally efficient OMM signals in accordance with channel status under an error rate constraint, constant-power adaptive OMM systems are shown to significantly outperform the conventional constant-power adaptive orthogonal frequency division multiplexing systems in average spectral efficiency.
\end{abstract}

Index Terms-Adaptive modulation, orthogonally multiplexed modulation, orthogonal frequency division multiplexing, spectral efficiency.

\section{INTRODUCTION}

$\mathbf{C}$ ONSTANT-POWER adaptive orthogonal frequency division multiplexing (AOFDM) [1]-[4] is adopted in practical wireless broadband communication systems [5]-[6] for its high average spectral efficiency and reliability. Typically, constant-power AOFDM systems partition the total band into subbands and temporally adapt the subcarrier modulation in each subband in the form of quadrature amplitude modulation (QAM) or phase shift keying (PSK) according to subband channel status in a way that a preassigned bit error rate (BER) is sustained. However, when a subband is so deeply faded that no QAM or PSK modulation is available to meet the BER requirement, no signal is transmitted over that subband and this signaling silence (commonly referred to as outage [7]) results in a waste of spectrum resource. Traditionally, error correcting codes [5]-[8] and/or antenna diversity [9] are used to provide improved robustness and thus reduce the proportion of time on signaling silence. This, however, trades off much higher implementation complexity. Without significantly trading off implementation complexity, other multicarrier modulation formats that provide more choices of power and spectral efficiencies than OFDM are considered in this letter to reduce the proportion of time on signaling silence and thus improve the average spectral efficiency.

Recently, four orthogonally multiplexed modulation (OMM) families, including orthogonally multiplexed orthogonal amplitude modulation (OMOAM) [10], orthogonally multiplexed orthogonal phase modulation (OMOPM) [11], orthogonally multiplexed on-off-keyed amplitude modulation $\left(\mathrm{OMO}^{2} \mathrm{AM}\right)$,

Manuscript received December 18, 2006; revised May 7, 2007 and July 5, 2007; accepted August 30, 2007. The associate editor coordinating the review of this paper and approving it for publication was C. Xiao. This work was supported by the ROC National Science Council under Contract 95-2221-E002-087-MY3

W.-L. Lin is with the Department of Electrical Engineering, National Central University, Chung-Li, Taiwan 32054, Republic of China (e-mail: s9521045@cc.ncu.edu.tw).

C.-D. Chung is with the Department of Electrical Engineering and the Graduate Institute of Communication Engineering, National Taiwan University, Taipei, Taiwan 10617, Republic of China (e-mail: cdchung@cc.ee.ntu.edu.tw). Digital Object Identifier 10.1109/TWC.2008.061082. and orthogonally multiplexed on-off-keyed phase modulation $\left(\mathrm{OMO}^{2} \mathrm{PM}\right)$ [12], have been defined to provide a multitude of new multidimensional modulations. Specifically, when constructed from a basis set of $2 N$ orthonormal basis signals, the OMM signal is generally expressed by multiplexing $M$ orthogonal and independent $(2 N / M)$-dimensional $((2 N / M)$ D) component signals. For OMOAM and OMOPM, the component signal is formed by grouping $L$ orthogonal pulseamplitude-modulated (for OMOAM) or phase-shift-keyed (for OMOPM) signals with amplitude or phase taking value in a $K$-ary alphabet. For $\mathrm{OMO}^{2} \mathrm{AM}$ and $\mathrm{OMO}^{2} \mathrm{PM}$, the component signal is constructed by multiplexing $L$ groups of modulated basis signals in which all basis signals in each group are on-off-keyed and further pulse-amplitude-modulated (for $\mathrm{OMO}^{2} \mathrm{AM}$ ) or phase-shift-keyed (for $\mathrm{OMO}^{2} \mathrm{PM}$ ) with amplitude or phase taking value in a $K$-ary alphabet. For simplicity, the modulation elements in each $2 N-\mathrm{D}$ OMM family can be conveniently indexed by the modulation parameter triplet $(M, L, K)$. By appropriately assigning multicarrier basis signal sets, both OMOAM and OMOPM families are shown in [10]-[11] to contain several conventional multicarrier modulations as special embodiments, including OFDM modulation. Moreover, the $2 N$-D OMOAM and OMOPM signals constructed from the same basis set exhibit the identical power spectral density (PSD), without regard to the modulation parameters or the basis signal assignment.

Among various OMOAM and OMOPM signals, OFDM is shown to provide the highest spectral efficiency, while there exists a multitude of modulation elements employing a smaller orthogonal multiplexing level that are more power efficient but slightly less spectrally efficient than OFDM. Unlike OMOAM and OMOPM, the power spectrum shapes of $\mathrm{OMO}^{2} \mathrm{AM}$ and $\mathrm{OMO}^{2} \mathrm{PM}$ constructed from spectrally overlapping basis signals depend strongly on basis signal assignment. When using the rectangularly-pulsed multicarrier basis set that constructs most OFDM signals, denoted by $\Omega_{2}$ in [10]-[12], some $\mathrm{OMO}^{2} \mathrm{AM}$ and $\mathrm{OMO}^{2} \mathrm{PM}$ signals constructed by grouping the quadrature carrier signals of adjacent frequencies together can even outperform certain OFDM signals in both power and spectral efficiencies [12]. In addition to the distinguished trends on spectral and error performance characteristics, the OMM signals constructed from $\Omega_{2}$ or the sinusoidally-pulsed basis set in [10]-[12] are also suited for fast Fourier transform (FFT) implementation. These merits motivate us to adopt the rectangularly-pulsed multicarrier $\mathrm{OMM}$ as the multidimensional modulation format in constant-power adaptive systems with an attempt to outperform AOFDM in average spectral efficiency, while allowing for standard OFDM-typed implementation. 


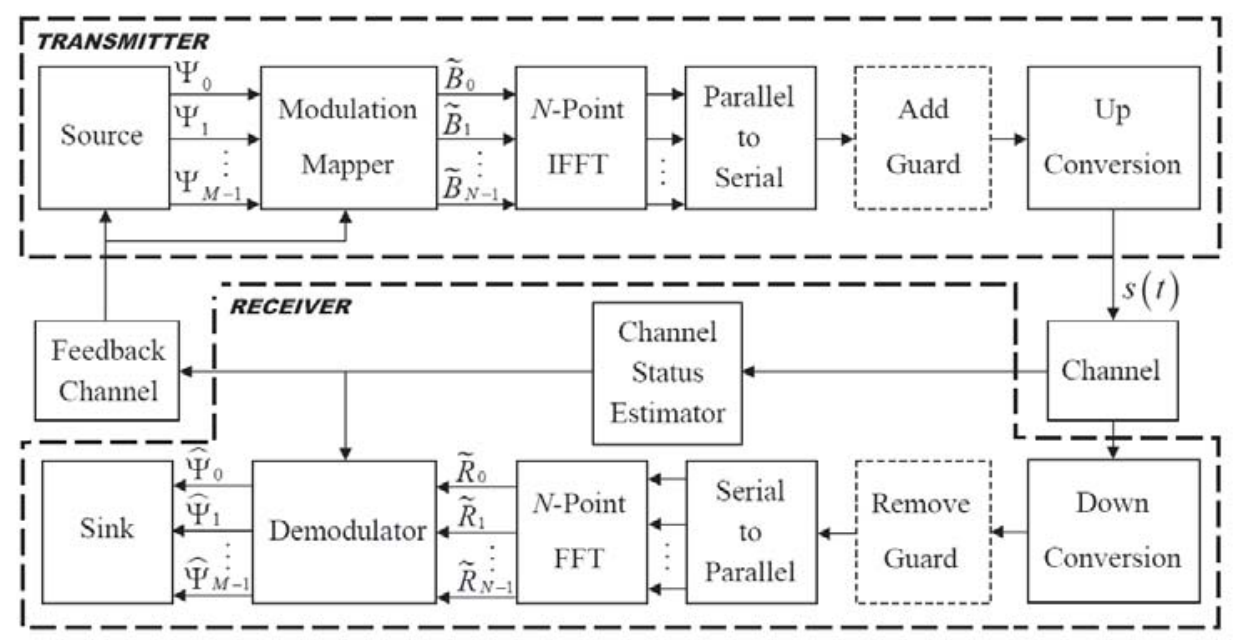

Fig. 1. The AOMM system model.

\section{SySTEM MODEL}

Fig. 1 depicts the considered $2 N-\mathrm{D}$ adaptive OMM (AOMM) system. In the nominal block interval of length $T$ seconds, the source generates $M$ independent and memoryless supersymbol streams simultaneously, with the $m$-th supersymbol denoted by $\Psi_{m} \triangleq\left[a_{m}, b_{m}^{(0)}, b_{m}^{(1)}, \ldots, b_{m}^{(L-1)}\right], m \in \mathcal{Z}_{M}$, where $a_{m}$ and $\left\{b_{m}^{(l)} \mid l \in \mathcal{Z}_{L}\right\}$ represent the embedded data symbols that are used to determine the signaling subcarriers and subcarrier modulations, respectively. ${ }^{1}$ The symbol alphabets for $b_{m}^{(l)}$ 's and $a_{m}$ are given by $b_{m}^{(l)} \in \mathcal{Z}_{K}, a_{m} \in \mathcal{Z}_{N_{x} / L}$ for OMOAM and OMOPM, and $a_{m} \in\left\{1,2, \ldots, 2^{N_{x} / L}-1\right\}$ for $\mathrm{OMO}^{2} \mathrm{AM}$ and $\mathrm{OMO}^{2} \mathrm{PM}$. Here, the parameter $N_{x}$ is defined by $N_{x}=N_{a} \triangleq 2 N / M$ for OMOAM and $\mathrm{OMO}^{2} \mathrm{AM}$ and $N_{x}=N_{p} \triangleq N / M$ for OMOPM and $\mathrm{OMO}^{2} \mathrm{PM}$. These $M$ supersymbols $\left\{\Psi_{m} \mid m \in \mathcal{Z}_{M}\right\}$ are then transformed by the modulation mapper to $N$ transmitted complex symbols $\left\{\widetilde{B}_{n} \mid n \in \mathcal{Z}_{N}\right\}$ according to the mapping rule in Table I where different rules are defined for different OMM families. By monitoring the channel information fed back from the receiver, both source and modulation mapper operate adaptively so that appropriate OMM signals are used in accordance with channel status. After the mapper, $\left\{\widetilde{B}_{n} \mid n \in \mathcal{Z}_{N}\right\}$ are modulated in parallel with $N$ subcarriers, uniformly spaced by $\omega_{d} \triangleq 2 \pi / T$, and multiplexed to form the transmitted OMM signal as [10][12]

$$
s(t)=\rho \sqrt{\frac{P T}{M L}} \operatorname{Re}\left\{\sum_{n=0}^{N-1} \widetilde{B}_{n} \exp \left(j\left(\omega_{0}+n \omega_{d}\right) t\right)\right\}
$$

for the nominal block interval. $P$ is the signal transmission power. $\omega_{0}$ is the reference frequency with $\omega_{0} \gg$ $\omega_{d}$. The factor $\rho$ is given by $\beta, 1, \kappa_{a} \beta$, and $\kappa_{p}$ for OMOAM, OMOPM, $\mathrm{OMO}^{2} \mathrm{AM}$, and $\mathrm{OMO}^{2} \mathrm{PM}$, respectively, where we have defined $\beta \triangleq\left[\left(K^{2}-1\right) / 3\right]^{-1 / 2}$ and $\kappa_{x} \triangleq$ $\left[\left(N_{x} / L\right) 2^{N_{x} / L-1} /\left(2^{N_{x} / L}-1\right)\right]^{-1 / 2}$. Note that the signal in the form of (1) can be efficiently realized by an inverse FFT

\footnotetext{
${ }^{1}$ Throughout this letter, $\mathcal{Z}_{I} \triangleq\{0,1, \ldots, I-1\}$.
}

(IFFT) architecture as depicted in Fig. 1 and also permits the insertion of guard intervals with cyclic prefix or zero padding to reject intersymbol interference (ISI) in the dispersive channels [13]. ${ }^{2}$ The guard insertion is, however, not treated herein in that the considered flat fading channel does not introduce ISI.

For design convenience, the parameters $N, M, L$ and $N_{x}$ are constrained throughout to be nonnegative powers of two. Such constraint is not prerequisite for general OMM signaling formats, though [10]-[12].

When realized in an IFFT architecture, the AOMM transmitter differs from the AOFDM transmitter only in the use of the modulation mapper. As depicted in Table I, these modulation mappers can be digitally realized without using any real multiplication. ${ }^{3}$

In this letter, AOMM with a small $N$ (the number of subcarriers) is considered for design and performance in flat fading. Although not treated explicitly, the results can also be applied to AOMM with a large number of subcarriers in the frequency-selective fading channel wherein the total band is modeled as a partition of subbands in a way that all subcarriers in a subband suffer the same fade. This subband flat fading model is plausible when the coherence bandwidth of the frequency-selective fading channel exceeds the bandwidth of a subband and commonly assumed for the study in AOFDM systems [1]-[2], [4].

Assume that the received signal is perfectly synchronized in amplitude, phase, symbol timing, and frequency by the receiver. As shown in Fig. 1, the received signal can be demodulated by the standard FFT-based OFDM demodulation process to yield $\left\{\widetilde{R}_{n} \mid n \in \mathcal{Z}_{N}\right\}$. Here, $\widetilde{R}_{n}$ is the received complex symbol on the $n$-th subcarrier, defined by $\widetilde{R}_{n}=$ $\rho \sqrt{g P T /(M L)} \widetilde{B}_{n}+\widetilde{W}_{n}$ where $\sqrt{g}$ is the flat fading gain that is assumed to remain constant within the nominal block

\footnotetext{
${ }^{2}$ In the case with guard insertion, the subcarrier spacing $\omega_{d}$ has to be redefined by $\omega_{d}=2 \pi / T_{d}$ with $T_{d}$ being the length of useful data interval.

${ }^{3}$ The computations concerning the data symbols $\left\{b_{m}^{(l)} \mid m \in \mathcal{Z}_{M}, l \in \mathcal{Z}_{L}\right\}$, i.e., $\left(K-1-2 b_{m}^{(l)}\right)$ and $\exp \left(j 2 \pi b_{m}^{(l)} / K\right)$, are assumed to be prestored.
} 
TABLE I

THE MODULATION MAPPING RULES AND DECISION ALGORITHMS OF FOUR $2 N$-D $(M, L, K)$ OMM FAMILIES. FOR OMOAM AND OMO ${ }^{2}$ AM, WE DENOTE $\widetilde{B}_{n}=B_{2 n}+j B_{2 n+1}$ AND $\widetilde{R}_{n}=R_{2 n}+j R_{2 n+1}$ WITH $B_{n}$ 'S AND $R_{n}$ 'S BEING REAL. FOR OMO ${ }^{2}$ AM AND OMO ${ }^{2}$ PM, BINARY EXPANSION $a_{m}=\sum_{i=0}^{N_{x} / L-1} a_{m}^{(i)} 2^{i}$ IS EMPLOYED FOR $m \in \mathcal{Z}_{M}$ WHERE $\left\{a_{m}^{(i)} \in \mathcal{Z}_{2} \mid i \in \mathcal{Z}_{N_{x} / L}\right\}$ DETERMINES THE ON-OFF-KEYING OF THE SUBCARRIERS.

\begin{tabular}{|c|c|c|}
\hline $\begin{array}{l}\text { Modulation } \\
\text { Family }\end{array}$ & $\begin{array}{c}\text { Modulation } \\
\text { Mapping Rule }\end{array}$ & $\begin{array}{c}\text { Decision } \\
\text { Algorithm } \\
\end{array}$ \\
\hline OMOAM & $B_{n}= \begin{cases}K-1-2 b_{m}^{(l)}, & \text { if } n=m N_{a}+l N_{a} / L+a_{m} \\
m \in \mathcal{Z}_{M}, l \in \mathcal{Z}_{L} \\
\text { otherwise }\end{cases}$ & $\begin{aligned} \widehat{\Psi}_{m} & =\arg \left\{\begin{array}{l}\max _{a_{m}} \\
\sum_{l=0}^{L-1} \max \\
l b_{m}^{(l)}\end{array}\left\{\left(K-1-2 b_{m}^{(l)}\right) R_{m N_{a}+l N_{a} / L+a_{m}}\right.\right. \\
- & \left.\left.\beta \sqrt{\frac{g P T}{4 M L}}\left(K-1-2 b_{m}^{(l)}\right)^{2}\right\}\right\}\end{aligned}$ \\
\hline ОМОРМ & $\widetilde{B}_{n}= \begin{cases}\exp \left(j \frac{2 \pi}{K} b_{m}^{(l)}\right), & \text { if } n=m N_{p}+l N_{p} / L+a_{m} \\
& \begin{array}{c}m \in \mathcal{Z}_{M}, l \in \mathcal{Z}_{L} \\
\text { otherwise }\end{array}\end{cases}$ & 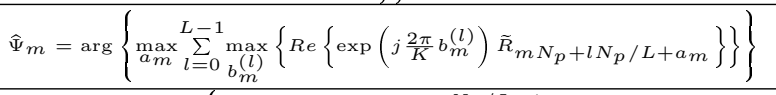 \\
\hline $\mathrm{OMO}^{2} \mathrm{AM}$ & $B_{n}= \begin{cases}a_{m}^{(i)}\left(K-1-2 b_{m}^{(l)}\right), & \begin{array}{c}\text { if } n=m N_{a}+l N_{a} / L+i, \\
m \in \mathcal{Z}_{M}, l \in \mathcal{Z}_{L}, i \in \mathcal{Z}_{N_{a} / L} \\
\text { otherwise }\end{array} \\
0, & \end{cases}$ & 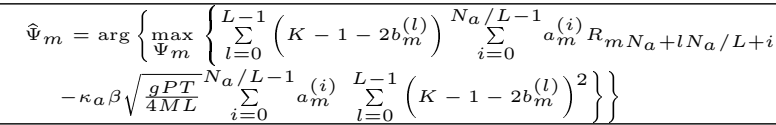 \\
\hline $\mathrm{OMO}^{2} \mathrm{PM}$ & $\widetilde{B}_{n}= \begin{cases}a_{m}^{(i)} \exp \left(j \frac{2 \pi}{K} b_{m}^{(l)}\right), & \text { if } n=m N_{p}+l N_{p} / L+i, \\
& m \in \mathcal{Z}_{M}, l \in \mathcal{Z}_{L}, i \in \mathcal{Z}_{N_{p} / L} \\
0, & \text { otherwise }\end{cases}$ & 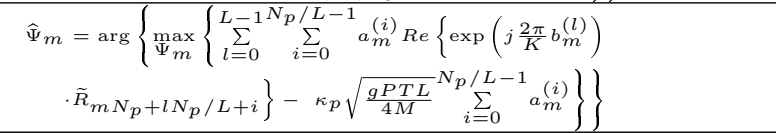 \\
\hline
\end{tabular}

TABLE II

COMPLEXITY COMPARISON OF THE MAXIMUM-LIKELIHOOD DECISION ALGORITHMS AMONG $2 N$-D $(M, L, K)$ OMM FAMILIES AND OFDM. THE COMPUtATIONS OF $\beta \sqrt{\frac{P T}{4 M L}}\left(K-1-2 b_{m}^{(l)}\right)^{2}, \kappa_{a} \beta \sqrt{\frac{P T}{4 M L}} \sum_{i=0}^{N_{a} / L-1} a_{m}^{(i)} \sum_{l=0}^{L-1}\left(K-1-2 b_{m}^{(l)}\right)^{2}$, AND $\kappa_{p} \sqrt{\frac{P T L}{4 M}} \sum_{i=0}^{N_{p} / L-1} a_{m}^{(i)}$ THAT ARE REQUIRED FOR OMM DECISION ALGORITHMS ARE ASSUMED PRESTORED AND NOT COUNTED IN EVALUATING THE NUMBERS OF REAL MULTIPLICATIONS AND REAL ADDITIONS.

\begin{tabular}{c|c|c}
\hline \hline $\begin{array}{c}\text { Maximum-Likelihood } \\
\text { Decision Algorithm }\end{array}$ & $\begin{array}{c}\text { Number of Real } \\
\text { Multiplications/T }\end{array}$ & $\begin{array}{c}\text { Number of Real } \\
\text { Additions/T }\end{array}$ \\
\hline \hline OMOAM & $O(4 N K)$ & $O\left(2 N\left(K+1-L^{-1}\right)\right)$ \\
\hline OMOPM & $O(4 N K)$ & $O\left(N\left(K+1-L^{-1}\right)\right)$ \\
\hline $\mathrm{OMO}^{2} \mathrm{AM}$ & $O\left((2 N+M L+M)\left(2^{N_{a} / L}-1\right) K^{L}\right)$ & $O\left(2 N\left(2^{N_{a} / L}-1\right) K^{L}\right)$ \\
\hline $\mathrm{OMO}^{2} \mathrm{PM}$ & $O\left((3 N+M)\left(2^{N_{p} / L}-1\right) K^{L}\right)$ & $O\left(N\left(2^{N_{p} / L}-1\right) K^{L}\right)$ \\
\hline OFDM $/ K^{2}$-QAM & $O(4 N K)$ & $O(2 N K)$ \\
\hline OFDM $/ K$-PSK & $O(4 N K)$ & $O(N K)$ \\
\hline
\end{tabular}

interval and $\left\{\widetilde{W}_{n} \mid n \in \mathcal{Z}_{N}\right\}$ are the circularly symmetric complex Gaussian noise (AWGN) samples which are independent and identically distributed with zero mean and variance $E\left\{\left|\widetilde{W}_{n}\right|^{2}\right\}=N_{0}$. By monitoring $\left\{\widetilde{R}_{n} \mid n \in \mathcal{Z}_{N}\right\}$, the channel status estimator estimates the instantaneously received signal-to-noise power ratio (SNR) $\gamma \triangleq g P T / N_{0}$ and pass the estimate to the decision algorithm in the receiver and also to the transmitter through an ideal error-free feedback channel. When $\gamma$ is perfectly measured by the channel status estimator, the maximum-likelihood decision algorithms that have been designed in [10]-[12] for the AWGN channel can be directly applied on $\left\{\widetilde{R}_{n} \mid n \in \mathcal{Z}_{N}\right\}$, as summarized in Table I, to determine the detected supersymbols $\left\{\widehat{\Psi}_{m} \mid m \in \mathcal{Z}_{M}\right\}$. The complexities required for digitally implementing such decision algorithms are given in Table II. As indicated, the complexities required by OMM are only marginally higher than the complexity required by OFDM.

\section{AdAPTATION SCHEME}

Denote $\Lambda \triangleq\left\{\lambda_{q} \mid q \in \mathcal{Z}_{Q}\right\}$ as a set of $Q 2 N$-D OMM modulations where $\lambda_{q}$ represents a modulation element and is arranged in such an index order that $\lambda_{p}$ has higher power efficiency but lower spectral efficiency than $\lambda_{q}$ if $p<q$. Under the constraints that the transmission power is constant and that instantaneous BER is not larger than a preassigned value $B E R_{T}$, AOMM adaptively chooses a modulation from $\Lambda$ for transmission according to the value of $\gamma$ in a way that the average spectral efficiency $\eta$ over a long time period is maximized.

For a modulation element $\lambda_{q}$, the power efficiency $\Gamma_{\lambda_{q}}$ is defined by the SNR value required to achieve $B E R_{T}$, and the spectral efficiency $\Phi_{\lambda_{q}}$ is defined by the inverse of the normalized bandwidth $\left(C T_{b}\right)^{-1}$ required to capture a preassigned fraction $\wp$ of total power within a bandwidth $C$, with $T_{b}$ being the bit time. ${ }^{4}$ Therefore, $\lambda_{q}$ is more powerefficient as $\Gamma_{\lambda_{q}}$ is smaller and more spectrally efficient as $\Phi_{\lambda_{q}}$ is larger. Note that the analytical expressions of PSD's and BER upper bounds derived in [10]-[12] can be used to compute power and spectral efficiencies for OMM.

Because $\Gamma_{\lambda_{p}}<\Gamma_{\lambda_{q}}$ and $\Phi_{\lambda_{p}}<\Phi_{\lambda_{q}}$ for $p<q$, a constant-power AOMM system based on $\Lambda$ transmits $\lambda_{q}$ when $\gamma \in\left[\Gamma_{\lambda_{q}}, \Gamma_{\lambda_{q+1}}\right)$ and sends no signal when $\gamma<\Gamma_{\lambda_{0}}$, with $\Gamma_{\lambda_{Q}}=\infty$ by default. This adaptation scheme yields the average spectral efficiency

\footnotetext{
${ }^{4}$ In the literature [1]-[4], [8]-[9], the spectral efficiency was commonly defined as the data bit rate and applicable when all modulation elements share the identical power spectrum shape. Such a definition does, however, not apply to the case herein since $\mathrm{OMO}^{2} \mathrm{AM}$ and $\mathrm{OMO}^{2} \mathrm{PM}$ signals have different spectrum shapes from those of OMOAM and OMOPM signals.
} 
TABLE III

THE MODULATION SETS FOR $\Lambda$ WITH THE ASSOCIATED POWER EFFICIENCY (IN DB) AND SPECTRAL EFFICIENCY (IN BITS/SEC/Hz) FOR $N=8$, $K_{\max }=16$ FOR OMOAM AND OMO ${ }^{2} \mathrm{AM}, K_{\max }=256$ FOR OMOPM AND OMO ${ }^{2} \mathrm{PM}, \wp=0.99$, AND $B E R_{T}=10^{-5}$. THE BOLDFACE TRIPLET ENTRIES CORRESPOND TO OFDM MODULATIONS. FOR OMO ${ }^{2}$ AM AND OMO ${ }^{2} \mathrm{PM}$, THE BASIS SIGNAL GROUPING RULE $E_{1}$ IN [12] IS USED FOR MODULATION ELEMENTS MARKED BY ASTERISK, WHILE THE RULE $S_{1}$ IN [12] IS ADOPTED FOR THE OTHER MODULATION ELEMENTS.

\begin{tabular}{|c|c|c|c|c|c|c|c|c|c|c|c|c|}
\hline \multirow[b]{2}{*}{$q$} & \multicolumn{3}{|c|}{ OMOAM } & \multicolumn{3}{|c|}{ OMOPM } & \multicolumn{3}{|c|}{$\mathrm{OMO}^{2} \mathrm{AM}$} & \multicolumn{3}{|c|}{$\mathrm{OMO}^{2} \mathrm{PM}$} \\
\hline & $\lambda_{q},(M, L, K)$ & $\Gamma_{\lambda_{q}}$ & $\Phi_{\lambda_{q}}$ & $\lambda_{q},(M, L, K)$ & $\Gamma_{\lambda_{q}}$ & $\Phi_{\lambda_{q}}$ & $\lambda_{q},(M, L, K)$ & $\Gamma_{\lambda_{q}}$ & $\Phi_{\lambda_{q}}$ & $\lambda_{q},(M, L, K)$ & $\Gamma_{\lambda_{q}}$ & $\Phi_{\lambda_{q}}$ \\
\hline 0 & $(1,1,2)$ & 13.71 & 0.23 & $(1,2,2)$ & 13.44 & 0.19 & $(1,4,2)^{*}$ & 18.67 & 0.65 & $(1,4,2)$ & 17.24 & 0.46 \\
\hline 1 & $(1,4,2)$ & 15.42 & 0.28 & $(1,1,4)$ & 13.71 & 0.23 & $(1,8,2)$ & 19.60 & 0.79 & $(1,2,4)^{*}$ & 18.94 & 0.65 \\
\hline 2 & $(2,1,2)$ & 16.45 & 0.37 & $(1,2,4)$ & 15.42 & 0.28 & $(2,4,2)$ & 19.72 & 0.92 & $(1,4,4)$ & 19.60 & 0.79 \\
\hline 3 & $(2,4,2)$ & 18.52 & 0.46 & $(2,1,4)$ & 16.45 & 0.37 & $(2,2,2)^{*}$ & 21.70 & 0.97 & $(2,2,4)$ & 19.85 & 0.92 \\
\hline 4 & $(4,1,2)$ & 19.14 & 0.56 & $(2,2,4)$ & 18.52 & 0.46 & $(4,2,2)$ & 22.41 & 1.18 & $(2,1,4)^{*}$ & 21.70 & 0.97 \\
\hline 5 & $(4,4,2)$ & 21.63 & 0.74 & $(4,1,4)$ & 19.14 & 0.56 & $(2,1,2)^{*}$ & 24.60 & 1.48 & $(4,1,4)$ & 22.41 & 1.18 \\
\hline 6 & $(2,4,4)$ & 25.42 & 0.83 & $(\mathbf{8}, \mathbf{1}, \mathbf{4})$ & 21.63 & 0.74 & $(4,1,2)^{*}$ & 24.80 & 1.62 & $(4,1,8)$ & 24.60 & 1.51 \\
\hline 7 & $(4,2,4)$ & 25.61 & 0.93 & $(8,1,8)$ & 26.78 & 1.11 & $(8,1,2)$ & 25.59 & 1.71 & $(4,1,16)$ & 30.36 & 1.84 \\
\hline 8 & $(8,1,4)$ & 28.29 & 1.11 & $(\mathbf{8}, \mathbf{1}, \mathbf{1 6})$ & 32.49 & 1.48 & $(4,2,4)$ & 28.38 & 1.84 & $(4,1,32)$ & 36.24 & 2.17 \\
\hline 9 & $(16,1,4)$ & 28.49 & 1.48 & $(8,1,32)$ & 38.36 & 1.85 & $(4,1,4)^{*}$ & 31.35 & 1.95 & $(4,1,64)$ & 42.18 & 2.51 \\
\hline 10 & $(16,1,8)$ & 34.60 & 2.22 & $(\mathbf{8}, \mathbf{1}, \mathbf{6 4})$ & 44.28 & 2.22 & $(8,1,4)$ & 32.05 & 2.37 & $(4,1,128)$ & 48.13 & 2.83 \\
\hline 11 & $(16,1,16)$ & 40.57 & 2.96 & $(8,1,128)$ & 50.22 & 2.59 & $(4,2,8)$ & 33.51 & 2.51 & $(4,1,256)$ & 54.09 & 3.16 \\
\hline 12 & - & - & - & $(8,1,256)$ & 56.17 & 2.96 & $(8,1,8)$ & 37.74 & 3.03 & - & - & - \\
\hline 13 & - & - & - & - & - & - & $(4,2,16)$ & 38.81 & 3.16 & - & - & - \\
\hline 14 & - & - & - & - & - & - & $(8,1,16)$ & 43.23 & 3.69 & - & - & - \\
\hline
\end{tabular}

$$
\eta=\sum_{q=0}^{Q-1} \Phi_{\lambda_{q}}\left[F_{\gamma}\left(\Gamma_{\lambda_{q+1}}\right)-F_{\gamma}\left(\Gamma_{\lambda_{q}}\right)\right] \quad \text { bits/sec/Hz }
$$

where $F_{\gamma}(\cdot)$ denotes the cumulative distribution function of $\gamma$. In this letter, $\sqrt{\gamma}$ is assumed to follow Rayleigh statistic and in this case

$$
F_{\gamma}(\Gamma)=1-\exp (-\Gamma / \bar{\gamma})
$$

with $\bar{\gamma}$ denoting the average SNR. It should be noted that the following arguments based on (2) can be easily generalized to other amplitude fading statistics.

Consider the modulation set $\Theta$ that contains all $2 N-\mathrm{D}$ $(M, L, K)$ modulations with $K \leq K_{\max }$ in a given OMM family. Given the preassigned system design values $B E R_{T}$ and $\wp$, the following rule is adopted to choose the modulation elements in $\Lambda$ from $\Theta$ :

Step 1: Initially, $\lambda_{Q-1}$ is chosen as the most spectrally efficient modulation in $\Theta$.

Step 2: When $\lambda_{Q-1}$ is determined, $\lambda_{Q-2}$ is chosen as the modulation that gives the highest spectral efficiency among all the modulations in $\Theta$ which are more power-efficient than $\lambda_{Q-1}$.

Step $m$ : In general, when $\lambda_{Q-1}, \lambda_{Q-2}, \ldots, \lambda_{Q-m}$ are determined, $\lambda_{Q-m-1}$ is chosen as the modulation that gives the highest spectral efficiency among all the modulations in $\Theta$ which are more power-efficient than $\lambda_{Q-m} \cdot{ }^{5}$ Repeat this step till all $Q$ modulations are found or $\Theta$ is exhausted.

When the set size of $\Theta$ is large enough, the above rule guarantees the existence of $\Lambda$ with a proper size $Q$. Given $\wp=$ 0.99 and $B E R_{T}=10^{-5}$, Table III gives an example list of $\Lambda$ for four OMM families with $N=8, K_{\max }=16$ for OMOAM

\footnotetext{
${ }^{5}$ In general, there may exist a modulation element that is more spectrally efficient than the chosen $\lambda_{Q-m-1}$ but less power-efficient than $\lambda_{Q-m}$. In this case, such a modulation is not chosen in that the BER constraint cannot be met for $\gamma<\Gamma_{\lambda_{Q-m}}$. Further, if there is more than one modulation element satisfying the rule for choosing $\lambda_{Q-m-1}$, any one among these modulation elements can be chosen.
}

and $\mathrm{OMO}^{2} \mathrm{AM}$, and $K_{\max }=256$ for $\mathrm{OMOPM}$ and $\mathrm{OMO}^{2} \mathrm{PM}$. The basis signal grouping rules $S_{1}$ and $E_{1}$ in [12] are adopted for both $\mathrm{OMO}^{2} \mathrm{AM}$ and $\mathrm{OMO}^{2} \mathrm{PM}$ signals with $N_{x} / L=2$ and $N_{x} / L \geq 4$, respectively, to achieve highest possible spectral efficiencies. Note that the sets for $\Lambda$ result from exhaustive search and thus yield different sizes. The efficiencies for $\Gamma_{\lambda_{p}}$ and $\Phi_{\lambda_{q}}$ that meet the system requirements $\wp=0.99$ and $B E R_{T}=10^{-5}$ are also listed in Table III. As indicated, there exists many OMM modulations other than OFDM that can be used for adaptation. This also implies that AOMM systems provide lower transmission outage than AOFDM.

The above process can also be applied to $\Theta$ that contains modulation elements from multiple OMM families. In this case, $\Lambda$ may contain modulation elements from different families. However, more complexity is required to integrate multiple OMM families into a system, in that different formats for modulation mapper and decision algorithm are used for different families (see Table I).

\section{NUMERICAL RESULTS}

Figs. 2 and 3 compare the average spectral efficiency characteristics among various AOMM and AOFDM using QAM (AOFDM/QAM) or PSK (AOFDM/PSK) as component modulations in the flat Rayleigh fading channel where $\Theta$ is constrained by $\wp=0.99, B E R_{T}=10^{-5}, K_{\max }=16$ for OFDM/QAM, OMOAM and $\mathrm{OMO}^{2} \mathrm{AM}$, and $K_{\max }=256$ for OFDM/PSK, OMOPM and $\mathrm{OMO}^{2} \mathrm{PM}$. The underlined modulation sets for $\Lambda$ and the associated efficiencies for $\Gamma_{\lambda_{q}}$ 's and $\Phi_{\lambda_{q}}$ 's are as listed in Table III. As shown in Fig. 2 with $N=8, \mathrm{AOMO}^{2} \mathrm{AM}$ significantly outperform AOMOAM and AOFDM/QAM when average SNR is high, whereas AOMOAM performs better than $\mathrm{AOMO}^{2} \mathrm{AM}$ and AOFDM/QAM when average SNR is low. Both trends can be justified in Table III. The former trend occurs because, in high $\bar{\gamma}$ regions, the $\mathrm{OMO}^{2} \mathrm{AM}$ family offers several modulations which are more efficient in both power and spectrum than the OMOAM family. The latter trend results from the other fact that the OMOAM family provides more power-efficient modulations 


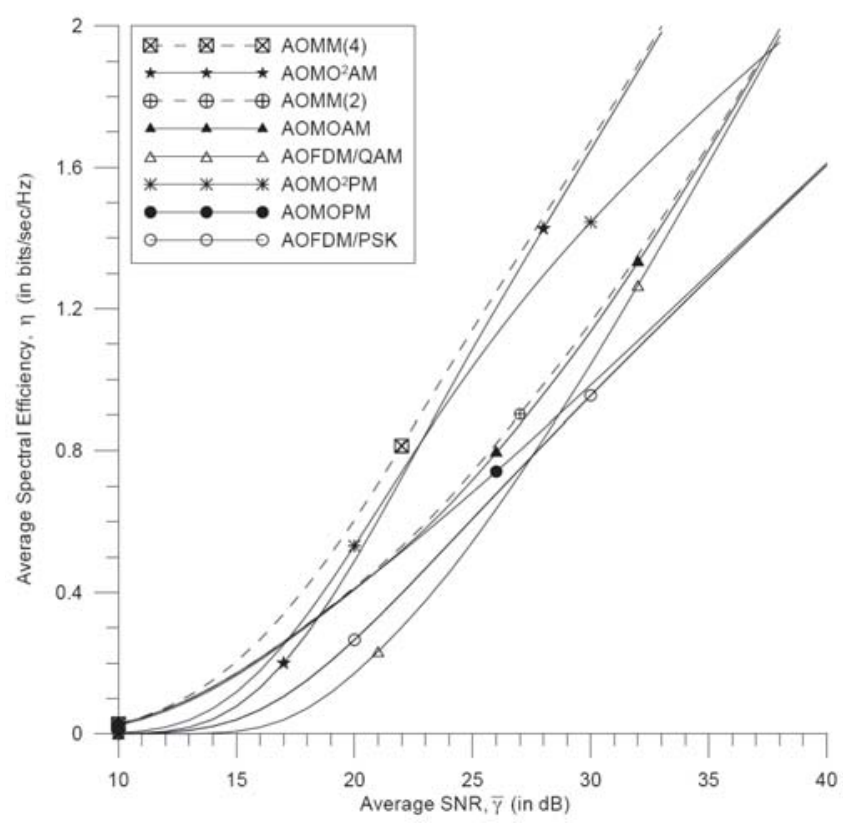

Fig. 2. Average spectral efficiency in flat Rayleigh fading channel for $N=8$, $K_{\max }=16$ for OMOAM and $\mathrm{OMO}^{2} \mathrm{AM}, K_{\max }=256$ for OMOPM and $\mathrm{OMO}^{2} \mathrm{PM}, \wp=0.99$, and $B E R_{T}=10^{-5}$.

in low $\bar{\gamma}$ regions. Similar performance trends can also be found among AOMOPM, AOMO ${ }^{2} \mathrm{PM}$, and AOFDM/PSK. As indicated in Fig. 2, both $\mathrm{AOMO}^{2} \mathrm{AM}$ and AOMOAM perform better than AOFDM/PSK and AOFDM/QAM for all values of $\bar{\gamma}$. Also shown in Fig. 2 are the results of AOMM systems based on $\Lambda$ obtained from OMOAM and OMOPM families, denoted by $\operatorname{AOMM}(2)$, and from OMOAM, $\mathrm{OMOPM}, \mathrm{OMO}^{2} \mathrm{AM}$, and $\mathrm{OMO}^{2} \mathrm{PM}$ families, denoted by $\operatorname{AOMM}(4)$. As indicated, $\operatorname{AOMM}(2)$ and $\operatorname{AOMM}(4)$ can provide marginal improvement in average spectral efficiency over $\mathrm{AOMOAM}$ and $\mathrm{AOMO}^{2} \mathrm{AM}$, respectively, at the cost of higher implementation complexities.

The average spectral efficiency characteristics for a smaller subband size $N=2$ are illustrated in Fig. 3. Similar performance trends as in Fig. 2 (with $N=8$ ) can be observed. However, the average spectral efficiencies achieved for $N=2$ are generally smaller than those achieved for $N=8$. This phenomenon is not unusual in that the OMM signals (e.g., OFDM signals) provide higher spectral compactness when a larger subband size is used. Therefore, a larger value for $N$ is desirable as long as the subband flat fading model holds good.

\section{CONCLUSION}

In this letter, the adaptive rectangularly-pulsed OMM systems are studied for the subband flat Rayleigh fading channel. The constant-power AOMM systems are shown to outperform the conventional constant-power AOFDM systems in average spectral efficiency, because more power efficient and spectrally efficient multidimensional modulation signals are adopted to increase system throughput. The considered AOMM systems permit FFT realization and guard insertion, and thus are suited for use in broadband applications where both implementation feasibility and system throughput are of paramount importance.

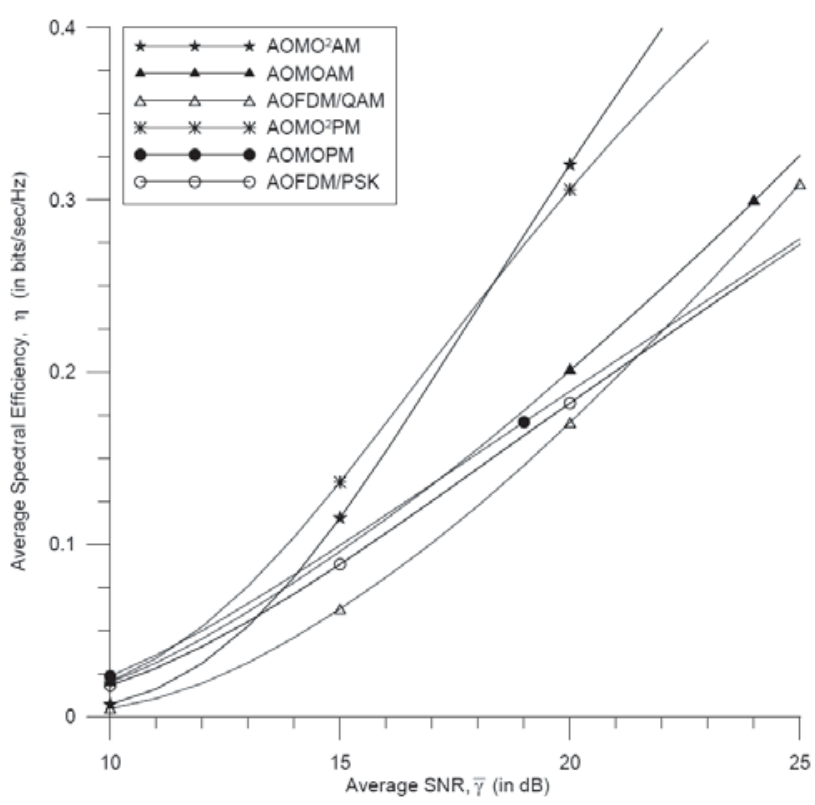

Fig. 3. Average spectral efficiency in flat Rayleigh fading channel for $N=2$, $K_{\max }=16$ for OMOAM and $\mathrm{OMO}^{2} \mathrm{AM}, K_{\max }=256$ for $\mathrm{OMOPM}$ and $\mathrm{OMO}^{2} \mathrm{PM}, \wp=0.99$, and $B E R_{T}=10^{-5}$.

\section{REFERENCES}

[1] T. Keller and L. Hanzo, "Adaptive modulation techniques for duplex OFDM transmission," IEEE Trans. Veh. Technol., vol. 49, pp. 18931906, Sept. 2000.

[2] B. Choi and L. Hanzo, "Optimum mode-switching-assisted constantpower single- and multicarrier adaptive modulation," IEEE Trans. Veh. Technol., vol. 52, pp. 536-560, May 2003.

[3] B. Canpolat and Y. Tanik, "Performance analysis of adaptive loading OFDM under Rayleigh fading," IEEE Trans. Veh. Technol., vol. 53, pp. 1105-1115, July 2004.

[4] S. Ye, R. S. Blum, and L. J. Cimini, "Adaptive modulation for variable rate OFDM systems with imperfect channel information," in Proc. IEEE Vehicular Technology Conf., Birmingham, AL, May 2002, pp. 767-771.

[5] ETSI, "Broadband Radio Access Networks (BRAN); HIPERLAN Type 2; Physical (PHY) layer," ETSI Std. TS 101475 V1.3.1, Dec. 2001.

[6] IEEE, "Local and Metropolitan Area Networks Part 16: Air Interface for Fixed Broadband Wireless Access Systems," IEEE Std. 802.16- 2004, Oct. 2004.

[7] M. J. Hossain, P. K. Vitthaladevuni, M.-S. Alouini, V. K. Bhargava, and A. J. Goldsmith, "Adaptive hierarchical modulation for simultaneous voice and multiclass data transmission over fading channels," IEEE Trans. Veh. Technol., vol. 55, pp. 1181-1194, July 2006.

[8] S. Vishwanath and A. J. Goldsmith, "Adaptive turbo-coded modulation for flat-fading channels," IEEE Trans. Commun., vol. 51, pp. 964-972, June 2003.

[9] T. Liew and L. Hanzo, "Space-time trellis and space-time block coding versus adaptive modulation and coding aided OFDM for wideband channels," IEEE Trans. Veh. Technol., vol. 55, pp. 173-187, Jan. 2006.

[10] C. D. Chung, "Orthogonally multiplexed orthogonal amplitude modulation family," IEEE Trans. Commun., vol. 50, pp. 415-428, Mar. 2002.

[11] — "Coherent and differentially coherent detections of orthogonally multiplexed orthogonal phase-modulated signals," IEEE Trans. Commun., vol. 51, pp. 428-440, Mar. 2003.

[12] C. D. Chung and H. S. Liaw, "Orthogonally multiplexed on-off-keyed amplitude and phase modulation families," IEEE Trans. Wireless Commun., vol. 4, pp. 288-299, Jan. 2005.

[13] B. Muquet, Z. Wang, G. B. Giannakis, M. de Courville, and P. Duhamel, "Cyclic prefixing or zero padding for wireless multicarrier transmissions?" IEEE Trans. Commun., vol. 50, pp. 2136-2148, Dec. 2002. 